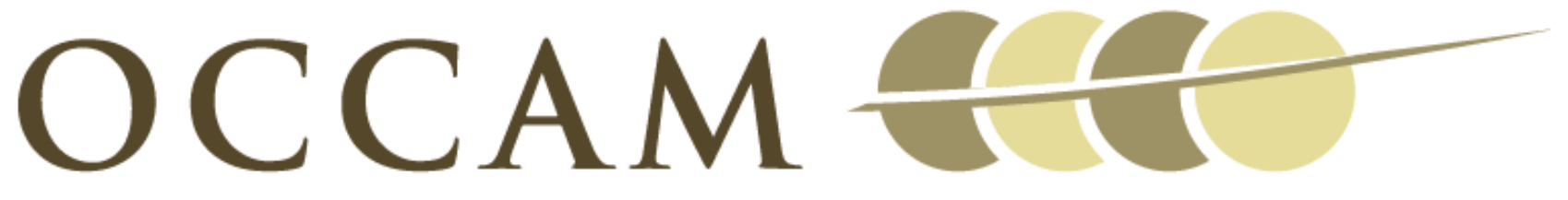

OXFORD CENTRE FOR COLLABORATIVE APPLIED MATHEMATICS

Report Number 10/59

Spin coating of an evaporating polymer solution

by

Andreas Munch, Colin P. Please, and Barbara Wagner

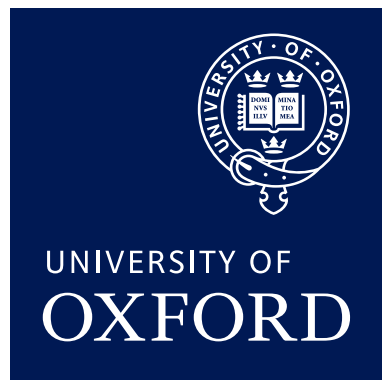

Oxford Centre for Collaborative Applied Mathematics Mathematical Institute

24 - 29 St Giles'

Oxford

OX1 3LB

England 



\section{Spin coating of an evaporating polymer solution}

Andreas Münch,, a) Colin P. Please, ${ }^{2, \text { b) }}$ and Barbara Wagner ${ }^{3, c)}$

1) Mathematical Institute, University of Oxford, 24-29 St. Giles', Oxford OX1 3LB, $U K$

2) School of Mathematics, University of Southampton, University Road, Southampton SO17 1BJ, UK

${ }^{3)}$ Weierstrass Institute of Applied Analysis and Stochastics, Mohrenstrasse 39, 10117 Berlin, Germany

We consider a mathematical model of spin coating of a single polymer blended in a solvent. The model describes the one-dimensional development of the thin layer of the mixture as the layer thins due to flow created by a balance of viscous forces and centrifugal forces and due to evaporation of the solvent. In the model both the diffusivity of the solvent in the polymer and the viscosity of the mixture are very rapidly varying functions of the solvent volume fraction. Guided by numerical solutions an asymptotic analysis reveals a number of different possible behaviours of the thinning layer dependent on the nondimensional parameters describing the system.

The main practical interest is in controlling the appearance and development of a "skin" on the polymer where the solvent concentration reduces rapidly on the outer surface leaving the bulk of the layer still with high concentrations of solvent. The critical parameters controlling this behaviour are found to be $\epsilon$ the ratio of the diffusion to advection time scales, $\delta$ the ratio of the evaporation to advection time scales and $\exp (-\gamma)$, the ratio of the diffusivity of the initial mixture and the pure polymer. In particular, our analysis shows that for very small evaporation with $\delta \ll \exp (-3 /(4 \gamma)) \epsilon^{3 / 4}$ skin formation can be prevented PACS numbers: 47.15.gm, 47.85.md, 61.25.he 66.20.Cy

Keywords: free boundary problem, asymptotic analysis, numerical simulation

\footnotetext{
a) Electronic mail: muench@maths.ox.ac.uk

b) Electronic mail: c.p.please@soton.ac.uk

c)Electronic mail: wagnerb@wias-berlin.de
} 


\section{INTRODUCTION}

Spin coating of polymers blended in volatile solvents is one of the most widespread methods used in the coating industry to produce a uniformly thin surface of as little as a few hundred nanometre thickness. It is used for many technologies including the production of electronic devices ${ }^{3}$, or organic solar cells ${ }^{7,8}$. One of the earliest theoretical studies goes back to Emslie et al. ${ }^{5}$. Further aspects, such as non-Newtonian rheology and colloidal suspensions were included by Acrivos et al. ${ }^{1}$ and Rehg et al. ${ }^{14}$.

For the applications mentioned above, evaporation of the solvent plays a crucial role for the evolution of the morphology. This has first been investigated experimentally by Kreith et al. ${ }^{9}$ and later by Birnie and Manley ${ }^{6}$. The first mathematical treatment of this aspect is due to Meyerhofer ${ }^{12}$ and was later extended by Sukanek ${ }^{17}$, Bornside et al. ${ }^{2}$ and Reisfeld et al. ${ }^{15,16}$. Due to the evaporation, the typically undesired phenomenon of skin formation may occur and has first been studied by Lawrence ${ }^{10,11}$, see also de Gennes ${ }^{4}$ and Okuzono et al. ${ }^{13}$ for further discussions.

Clearly, there are many time and spatial scales in this process and to our knowledge these have not been completely quantified. Moreover, this is the case even for the classic spin coating problem of a solution of a single polymer blended in a volatile solvent, which has been studied for many decades. Due to the growing technological interest in efficient organic solar cells it is of central importance to thoroughly understand the evaporative spin coating process and to be able to completely characterise and accurately describe it. With this aim in mind it is instructive to first consider the simple case of spin coating a single polymer solution with a volatile solvent and this is what we do in this paper. These ideas should motivate analysis of the more practical problem, where there are a blend with several polymer species and evaporation may lead to phase separation of the polymer mixture with formation and destabilisation of polymer-polymer interfaces. For the manufacturing of efficient organic solar cells for example, the sizes and morphologies of these interfaces need to be well controlled.

We base our study on the situation and experimental data given in Bornside et al. ${ }^{2}$. This process has several time scales which have been identified from numerical calculations. Roughly speaking, there is a very fast initial time scale lasting only a few seconds, which is

dominated by convection of fluid in radial direction accompanied by very fast thinning and 
negligible evaporation. Subsequently, on a longer time scale convection becomes negligible and the process is dominated by evaporation of the solvent controlled by diffusion. There are further longer time scales that lead eventually to formation of a "skin". However, for more volatile solvents or larger initial volume fraction of the polymer, skin formation may occur on a much shorter time scale.

Our aim in this study is to quantify in which parameter regimes the behaviour will occur. We present a systematic approach using matched asymptotic expansions in order to quantitatively characterise the various processes. Our asymptotic analysis is able to determine the various parameter regimes, in particular when skin formation occurs and on what time scale. We compare our analytic solutions to those of our numerical code that uses nonuniform grids in order to capture the self-similar approach toward blow-up in our mathematical model, i.e. skin formation.

\section{GENERAL BEHAVIOUR OF A SPUN COAT LAYER}

From the detailed analysis of the model presented in this paper we have found that in most physically relevant circumstances there are a number of different behaviours that can occur. In examining these we consider the physically relevant case where changes in diffusion coefficient due to changes in solvent concentration occur on the same scale as the changes in the viscosity. These behaviours are dependent primarily on the relative importance of three time scales in the problem and the initial volume ratio of polymer to solvent. The three timescales are i) the diffusion time of the solvent across the initial layer, ii) the time to advect across the initial layer due to the vertical velocity induced by the centrifugal and viscous forces, and iii) the time to evaporate solvent from the initial layer. The relative size of these time scales is readily captured by considering the parameters $\epsilon$, the ratio of the diffusion to advection time scales and $\delta$, the ratio of the evaporation to advection time scales both of which are very small in any practical situation and the initial volume ratio is described by $\gamma$ which is also relatively small. Our analysis shows that there are three main regimes of behaviour corresponding to

(i) $\delta \ll \epsilon^{3 / 4} \ll 1$ (small evaporation)

(ii) $\epsilon^{3 / 4} \ll \delta \ll \epsilon^{1 / 2} \ll 1$ (medium evaporation) 


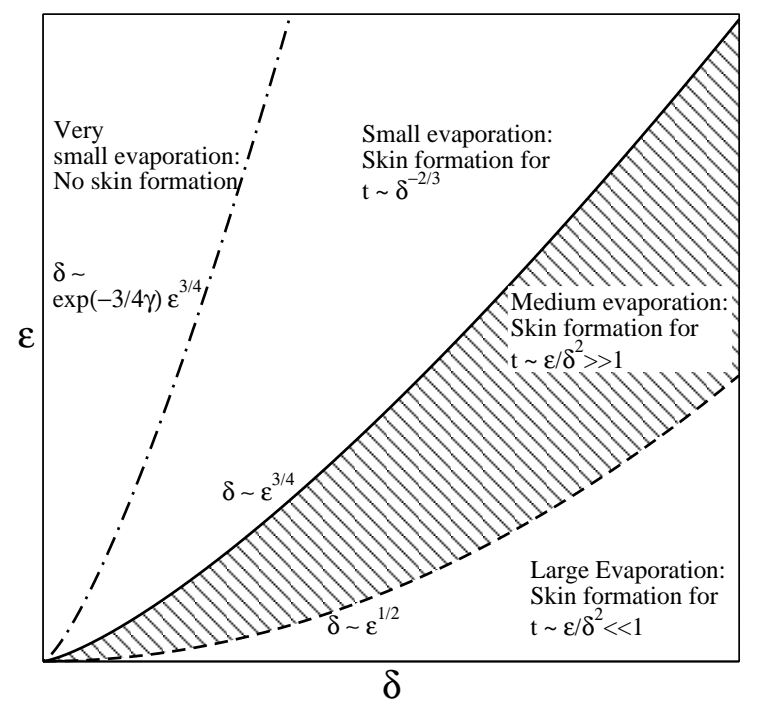

FIG. 1. Asymptotic regimes

(iii) $\epsilon^{1 / 2} \ll \delta \ll 1$ (large evaporation)

In each case the balance of mechanisms governing the development of the layer changes as time progresses. Most interestingly, the case (i) has a limiting case

(ia) $\delta \ll \exp (-3 /(4 \gamma)) \epsilon^{3 / 4}$ (very small evaporation)

where, unlike in the other cases, there is no skin formation.

In all cases initially the layer thins due to centrifugal forces balancing with viscous stresses while evaporation causes changes in solvent concentration in a narrow boundary layer near the surface. If the solvent volume fraction variations in this boundary layer becomes significant so that diffusion drops dramatically, a skin is formed. Once a skin has formed, there is a much longer time scale over which the volume fraction slowly equilibrates.

In the large evaporation case, the skin forms before the layer thins significantly; in the medium evaporation case, the skin forms after the layer has thinned. In both cases, when the thin skin forms, the material under the skin still has its initial consistency.

When the evaporation rate is small, a thin skin forms with the material underneath being spatially uniform but at a solvent concentration that is much less than its initial value. For the very small evaporation rate, no skin forms. 


\section{BASIC ONE-DIMENSIONAL MODEL}

We consider the movement of a mixture on a spinning disk which spins with angular velocity $\omega$ about the $z$ axis (hence $\boldsymbol{\omega}=\boldsymbol{\omega} \boldsymbol{z}$ ). The coordinate system is fixed in the disk with $r$ as the radial distance and the mixture between the spinning disk $z=0$ and the upper surface $z=h(r, t)$. The mixture is taken to have a volume fraction of the volatile solvent $\phi(r, z, t)$ with the polymer having volume fraction $1-\phi(r, z, t)$. We consider the mixture to flow radially symmetrically and act as an incompressible Newtonian fluid, with the fluid velocity being velocity $\boldsymbol{u}$ with components $u(r, z, t)$ in the radial direction and $w(r, z, t)$ in the $z$ direction. We assume the viscosity $\eta(\phi)$ to be very strongly dependent on the solvent volume fraction, and that diffusion of the solvent through the mixture be represented by a diffusion coefficient $\mathcal{D}(\phi)$, which is also very strongly dependent on the solvent volume fraction. We assume the density of the mixture, $\rho$, is independent of the solvent volume fraction so that the governing equations are as follows:

The momentum equations are

$$
\rho\left(\partial_{t} \boldsymbol{u}+\boldsymbol{u} \cdot \nabla \boldsymbol{u}\right)=\nabla \cdot \boldsymbol{T}-\rho[2 \boldsymbol{\omega} \times \boldsymbol{u}+\boldsymbol{\omega} \times(\boldsymbol{\omega} \times \boldsymbol{r})]
$$

where the stress tensor is given by $\boldsymbol{T}=-p \boldsymbol{I}+\eta(\phi) \dot{\gamma}$ with the strain rate $\dot{\gamma}=\nabla \boldsymbol{u}+(\nabla \boldsymbol{u})^{T}$ and $\boldsymbol{I}$ is the identity matrix. The continuity equation is given by

$$
\nabla \cdot \boldsymbol{u}=0
$$

These are coupled to the diffusion equation for the solvent volume fraction

$$
\partial_{t} \phi+\boldsymbol{u} \cdot \nabla \phi=\nabla \cdot(\mathcal{D}(\phi) \nabla \phi)
$$

where we chose for simplicity

$$
\eta(\phi)=\eta_{0} e^{-\phi / 2 b} \quad \text { and } \quad \mathcal{D}(\phi)=D_{0} e^{\phi / 2 a}
$$

for the functional dependency of the viscosity and diffusion on the volume fraction. The constants $\eta_{0}, D_{0}, a$ and $b$ are material constants. Note, that Bornside et al. ${ }^{2}$ used this exponential to describe the diffusion but used an algebraic expression to describe the viscosity. However, the precise details of the dependency of the diffusion coefficient and the viscosity are not essential to the following discussions. 
For the boundary conditions at the free surface $z=h(r, t)$ we have the normal and tangential stress conditions. We assume that there is a surface tension, $\sigma$, acting and no tangential stress so that

$$
\boldsymbol{n} \cdot \boldsymbol{T} \cdot \boldsymbol{n}=\sigma \nabla \cdot \mathbf{n} \quad \text { and } \quad \boldsymbol{n} \cdot \boldsymbol{T} \cdot \boldsymbol{t}=0
$$

For the kinematic condition we have

$$
\boldsymbol{u} \cdot \boldsymbol{n}-\frac{\partial_{t} h}{\sqrt{1+\partial_{r}^{2} h}}=\frac{J(\phi)}{\rho}
$$

where $J(\phi)$ denotes the flux due to evaporation.

At this surface conservation of solvent gives

$$
-\mathcal{D}(\phi) \nabla \phi \cdot \boldsymbol{n}+\phi\left(\boldsymbol{u} \cdot \boldsymbol{n}-\frac{\partial_{t} h}{\sqrt{1+\partial_{r}^{2} h}}\right) \rho=J(\phi)
$$

or, using (1f)

$$
\mathcal{D}(\phi) \nabla \phi \cdot \boldsymbol{n}=-J(\phi)(1-\phi)
$$

Following Bornside et $\mathrm{al}^{2}$ we take the empirical law

$$
J(\phi)=k\left(\phi-\phi_{1}\right)
$$

where $\phi_{1}$ is the equilibrium solvent volume fraction determined by conditions in the adjacent gas and $k$ is the mass transfer coefficient. Boundary conditions at the solid substrate $z=0$ are the no-slip and impermeability conditions

$$
\boldsymbol{u}=0 \quad \nabla \phi \cdot \boldsymbol{n}=0
$$

For the initial conditions we let

$$
h(r, 0)=h_{0} \quad \text { and } \quad \phi(r, z, 0)=\phi_{0} .
$$

To analyse the problem we put it into nondimensional form and consider suitable limiting cases from the various parameters in the problem. Note, we nondimensionalise the problem 
using the overbar notation for the nondimensional variables, but for simplicity of notation, we will immediately drop the overbar notation from thereon.

$$
\begin{gathered}
r=\mathbb{L} \bar{r}, \quad z=\mathbb{H} \bar{z}, \quad h=\mathbb{H} \bar{h}, \quad h_{0}=\mathbb{H}, \\
u=\mathbb{U} \bar{u}, \quad w=\varepsilon_{\ell} \mathbb{U} \bar{w}, \quad T=\frac{\mathbb{L}}{\mathbb{U}} \bar{t}
\end{gathered}
$$

where we take the characteristic height $\mathbb{H}$ as the initial height $h_{0}$ of the layer, $\mathbb{L}$ as the radius of the spinning disk, and introduce the aspect ratio $\varepsilon_{\ell}=\mathbb{H} / \mathbb{L}$. For the characteristic velocity we choose

$$
\mathbb{U}=\frac{2 \rho \omega^{2} \mathbb{H}^{2} \mathbb{L}}{\eta_{0} e^{-\phi_{0} / 2 b}}
$$

For $\phi$ we let

$$
\phi=\phi_{0}+2 a \bar{\phi}
$$

so that

$$
\eta=\eta_{0} e^{-\phi_{0} / 2 b} e^{-\mu \bar{\phi}} \text { and } \mathcal{D}=D_{0} e^{\phi_{0} / 2 a} e^{\bar{\phi}},
$$

where $\mu=a / b$. Notice that, because $a$ is rather small, this nondimensionalisation scales the solvent volume fraction to be very close to the initial value and this ensures we can easily see the strong dependency of the diffusivity and viscosity on the solvent.

For the rest of the paper we consider only the one-dimensional problem. This means we consider the case of uniform thickness of the liquid layer across the substrate, i.e. the free boundary $h$ is independent of $r$. In addition we assume that $\phi$ does not vary in the radial direction. We note that the assumption of a uniform thickness over the entire region is quite good except for neglecting variations with radius of the mass transfer rate to the surrounding gas, $k$.

For the thin liquid layer we make use of the fact that $\varepsilon_{\ell} \ll 1$ (and also that the reduced Reynolds number $\left(2 \rho^{2} \omega^{2} H^{4}\right) /\left(\eta_{0} \exp \left(-\phi_{0} /(2 b)\right)\right)$ is small). In the standard way the leading order lubrication approximation renders the pressure to be independent of $z$, so that integrating the radial component of the momentum equation twice w.r.t. $z$, using the leading order boundary conditions of tangential stress $\partial_{z} u=0$ and normal stress $p=0$ at the free 
boundary $z=h(t)$, and the no-slip $u=0$ and impermeability condition $w=0$ at the solid substrate $z=0$ one obtains the expression

$$
u(r, z, t)=\int_{0}^{z} r(h(t)-z) e^{\mu \phi} d z .
$$

Using the continuity equation and integrating w.r.t. $z$ we obtain

$$
w(z, t)=-\int_{0}^{z}(h(t)-q)(z-q) e^{\mu \phi} d q
$$

which couples to the nondimensionalised diffusion equation

$$
\partial_{t} \phi+w \partial_{z} \phi=\epsilon \partial_{z}\left(e^{\phi} \partial_{z} \phi\right)
$$

together with boundary conditions at $z=h(t)$

$$
\begin{aligned}
\frac{\epsilon}{\delta} e^{\phi} \partial_{z} \phi & =-\frac{1}{\beta}(1+\gamma \phi)(1-\beta \phi), \\
\partial_{t} h-w & =-\delta(1+\gamma \phi),
\end{aligned}
$$

the boundary conditions at $z=0$

$$
\partial_{z} \phi=0
$$

and the initial conditions

$$
h(0)=1 \quad \text { and } \quad \phi(z, 0)=0 .
$$

The resulting nondimensional parameters in the problem for $w(z, t), \phi(z, t)$ and $h(t)$ defined by (3a)-(3f) are given by

$$
\begin{aligned}
\epsilon & =\frac{1}{\varepsilon_{\ell} \mathrm{Pe}}, \delta=\frac{k\left(\phi_{0}-\phi_{1}\right)}{\varepsilon_{\ell} \mathbb{U}}, \text { where } \mathrm{Pe}=\frac{\mathbb{U} \mathbb{H}}{D_{0} e^{\phi_{0} / 2 a}}, \\
\gamma & =\frac{2 a}{\phi_{0}-\phi_{1}}, \quad \beta=\frac{2 a}{1-\phi_{0}} \quad \text { and } \quad \mu=\frac{a}{b} .
\end{aligned}
$$

Typical values for the constants, that are involved in the spin coating process are given e.g. in Bornside et al. ${ }^{2}$ and Kreith el al. ${ }^{9}$ :

$$
\begin{gathered}
D_{0}=7.8 \times 10^{-12} \mathrm{~cm}^{2} \mathrm{sec}^{-1}, \quad \mathbb{H}=10^{-2} \mathrm{~cm}, \\
\eta_{0}=1 \mathrm{P}, \quad \omega=3000 \mathrm{rad} \mathrm{sec}^{-1}, \quad \rho=1 \mathrm{~g} \mathrm{~cm}^{-3},
\end{gathered}
$$




$$
\begin{gathered}
\phi_{0}=0.9, \quad \phi_{1}=0, \quad a=1 / 20, \quad b=1 / 20, \\
k=4 \times 10^{-5}(\omega \mathrm{sec})^{1 / 2} \mathrm{~cm} \mathrm{sec}^{-1} .
\end{gathered}
$$

On the basis of these data, we find for the orders of magnitude of the nondimensional parameters

$$
\epsilon \approx 3.5 \times 10^{-7}, \quad \delta \approx 1.1 \times 10^{-4}, \quad \beta \approx 1, \quad \gamma \approx 0.1
$$

and the fact that the diffusivity and viscosity have very similar sensitivities to the solvent volume fraction implies $\mu \approx 1$. We note that this system has been considered by Reisfeld et al. ${ }^{15,16}$ but without the variability of the diffusivity or viscosity. For the analysis presented here, we shall assume from now on

$$
\epsilon \ll 1 \quad \delta \ll 1 \quad \text { and } \quad \gamma \ll 1 \text {, }
$$

that $\beta$ is of order one and $\mu$ is very near unity. The relative size of $\epsilon$ and $\delta$ will need to be considered as different cases.

Numerical solutions to this problem can readily be generated. Here, our aim is to find analytical expressions for the solutions by considering physically relevant limiting cases of the nondimensional parameters and hence gain insight into the range of values that give particular behaviour such as skin formation.

\section{ASYMPTOTIC REGIMES}

The behaviour of the mixture layer is different depending on the relative size of $\delta$ and $\epsilon$. We present the behaviour for three different regimes, starting from small evaporation rates, as this turns out the richest case and conclude with the regime for large evaporation rates.

\section{A. Small evaporation $\left(\delta \ll \epsilon^{3 / 4} \ll 1\right)$}

\section{1. $\quad$ Early time scale $(t=\mathcal{O}(1))$}

We start by considering the behaviour for $t=\mathcal{O}(1)$. In this regime the layer thins due to fluid flow alone and the solution has one behaviour in the bulk and another in a small diffusive boundary layer adjacent to the free surface. 
a. Behaviour in the bulk Taking the lowest order problem we find

$$
\begin{aligned}
& \partial_{t} \phi+w \partial_{z} \phi=0, \\
& w=-\int_{0}^{z}(z-q)(h(t)-q) e^{\mu \phi(q, t)} d q \\
& \partial_{t} h-w=0 \text { at } z=h \\
& h=1 \text { and } \phi=0 \text { at } t=0 .
\end{aligned}
$$

This has the solution

$$
\phi=0, \text { so that } w(z, t)=-\frac{h z^{2}}{2}+\frac{z^{3}}{6}
$$

with the condition at $z=h$, giving

$$
\partial_{t} h+\frac{h^{3}}{3}=0 \text { and hence } h(t)=\frac{1}{\sqrt{1+2 t / 3}} .
$$

This solution is well known but note that care needs to be taken in getting this solution as (5b) contains an integral over the whole region including the boundary layer and (5c) is imposed in the boundary layer, however, so long as the integrand never gets large in the boundary layer, which will be true in the limit here, this solution is correct to lowest order.

b. Behaviour in the diffusion boundary layer The boundary layer scalings are

$$
z=h(t)+\epsilon^{1 / 2} \bar{z} \quad \text { and } \quad \phi(z, t)=\frac{\delta}{\epsilon^{1 / 2}} \bar{\phi}(\hat{z}, t)
$$

and note, for small evaporation, this corresponds to $\phi$ remaining small in this region. The problem in the boundary layer is then

$$
\begin{gathered}
\partial_{t} \bar{\phi}-\frac{h^{2} \bar{z}}{2} \partial_{\bar{z}} \bar{\phi}=\partial_{\bar{z} \hat{z}} \bar{\phi}, \\
\text { at } \bar{z}=0 \partial_{\bar{z}} \bar{\phi}=-\frac{1}{\beta}, \\
\text { far field } \quad \bar{\phi} \rightarrow 0 \quad \text { as } \quad \bar{z} \rightarrow-\infty \\
\bar{\phi}=0 \quad t=0 .
\end{gathered}
$$

We note that, because of the form of $h(t)$ in $(7)$, after an initial transient to account for the initial condition, this problem has self-similar behaviour of the form

$$
\hat{\phi}=\frac{1}{\beta}\left(1+\frac{2}{3} t\right)^{1 / 2} u(\zeta), \quad \hat{z}=-\left(1+\frac{2}{3} t\right)^{1 / 2} \zeta
$$


described by the boundary value problem

$$
\begin{aligned}
& \frac{d^{2} u}{d \zeta^{2}}=\frac{1}{3} u-\frac{5}{6} \zeta \frac{d u}{d \zeta}, \\
& \frac{d u}{d \zeta}(0)=1, \\
& u \rightarrow 0 \text { as } \eta \rightarrow \infty .
\end{aligned}
$$

The solution to (11) can be written in terms of Kummer's functions

$$
\begin{aligned}
M(a, b, z) & :=\frac{\Gamma(b)}{\Gamma(a) \Gamma(b-a)} \int_{0}^{1} e^{z x} x^{a-1}(1-x)^{b-a-1} d x, \\
U(a, b, z) & :=\frac{1}{\Gamma(a)} \int_{0}^{\infty} e^{-z x} x^{a-1}(1+x)^{b-a-1} d x,
\end{aligned}
$$

and is given by

$$
\begin{aligned}
u(\zeta)= & {\left[1-\kappa \frac{2}{5} U\left(\frac{6}{5}, \frac{3}{2}, 0\right)+2 \kappa U\left(\frac{1}{5}, \frac{3}{2}, 0\right)\right] } \\
& \times e^{5 / 12 \zeta^{2}} M\left(\frac{6}{5}, \frac{3}{2}, \frac{5}{12} \zeta^{2}\right) \zeta \\
& +\kappa e^{5 / 12 \eta^{2}} U\left(\frac{6}{5}, \frac{3}{2}, \frac{5}{12} \zeta^{2}\right) \zeta .
\end{aligned}
$$

where we must take $\kappa=-0.366172$ for this to match to the outer solution $\phi=0$.

Comparison of this solution for $t=\mathcal{O}(1)$ with our numerical results are shown in figure 2 and indicates where the approximation break down in time.

\section{Medium time scale $\left(t=\mathcal{O}\left(\epsilon^{-1 / 2}\right)\right)$}

The previous results have established that as $t$ gets larger the layer thins according to $h=\mathcal{O}\left(t^{-1 / 2}\right)$ (from $\left.(7)\right)$ and, using (10), a boundary layer forms of thickness $\mathcal{O}\left((\epsilon t)^{1 / 2}\right)$ in which the volume fraction is of size $\phi=\mathcal{O}\left(\delta(t / \epsilon)^{1 / 2}\right)$. There are several scenarios how this solution ceases to be valid as time progresses. The possibilities are:

a) Evaporation eventually becomes important, so that

$$
\partial_{t} h+\frac{1}{3} h^{3}=\mathcal{O}(\delta) .
$$

This occurs when $t^{-3 / 2}=\mathcal{O}(\delta)$, i.e. on the time scale $t_{a}=\mathcal{O}\left(\delta^{-2 / 3}\right)$.

b) The thickness of the boundary layer grows to the size of the entire layer, i.e. when $h=\mathcal{O}\left((\epsilon t)^{1 / 2}\right)$. This occurs on the time scale $t_{b}=\mathcal{O}\left(\epsilon^{-1 / 2}\right)$. 


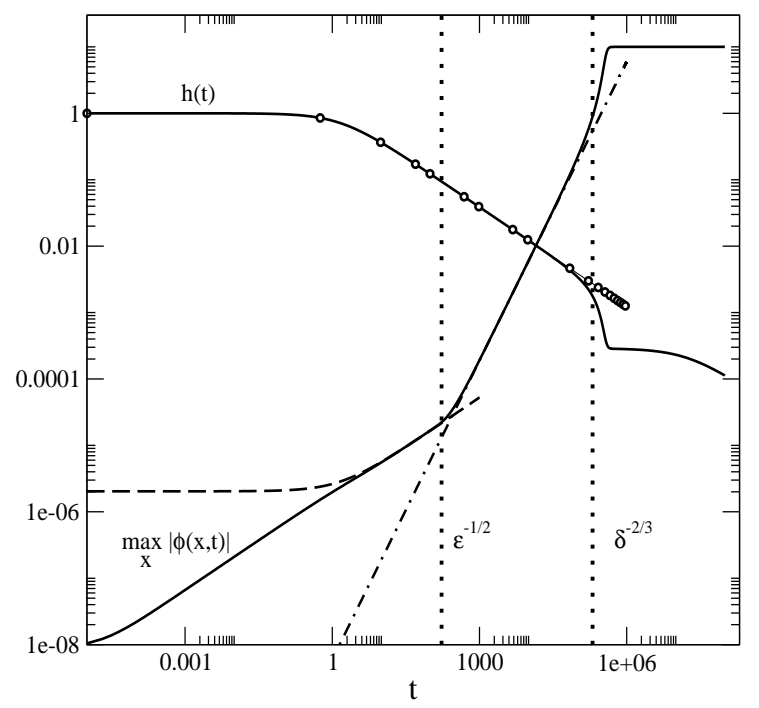

FIG. 2. Comparison of the numerical and asymptotic results in the small evaporation regime, for $\epsilon=3.5 \times 10^{-5}, \delta=1.1 \times 10^{-8}$, and for constant viscosity $\mu=0$. The solid curves denote the numerical results for $h(t)$ and $\max _{x}|\phi(x, t)|$ for (3). The dashed line shows $\max _{x}|\phi(x, t)|$ for the self-similar solution (10), (11), to the early time asymptotic problem; the behaviour for $h$, given by (7), is indicated by circles. The dash-dotted curves show the longtime approximations (17) for $\max _{x}|\phi(x, t)|$ for the leading order asymptotic solution in the medium time regime; note that in this regime, the leading order solution for $h(t)$ coincides with the long time expansion of (7), so we do not include a separate line. The two vertical dotted lines correspond to the times $t=\epsilon^{-1 / 2}=169$ and $t=\delta^{-2 / 3}=2.02 \times 10^{5}$, respectively.

c) Thirdly, the volume fraction $\phi$ increases to be $\mathcal{O}(1)$ in the boundary layer, resulting in formation of a skin with signficant variations in the diffusive and the viscosity. This occurs when $\delta(t / \epsilon)^{1 / 2}=\mathcal{O}(1)$, i.e. if $t_{c}=\mathcal{O}\left(\epsilon / \delta^{2}\right)$.

We note that there is one distinguished limit, where all three time scales, $t_{a}, t_{b}$ and $t_{c}$ are equal. This happens when $\delta \sim \epsilon^{3 / 4}$. In the case when $\delta \gg \epsilon^{3 / 4}$, which we will refer to as medium evaporation, the time scales are ordered according to $t_{c} \ll t_{a} \ll t_{b}$ and therefore skin formation occurs first. In the other case, when $\delta \ll \epsilon^{3 / 4}$, which we call small evaporation, the time scales are ordered according to $t_{b} \ll t_{a} \ll t_{c}$. We now study the small evaporation case and determine the behaviour on the timescale of $t=t_{b}=\mathcal{O}\left(\epsilon^{-1 / 2}\right)$.

Since $h=\mathcal{O}\left(t^{-1 / 2}\right)$ for large $t$, this shows that we should consider $h=\mathcal{O}\left(\epsilon^{1 / 4}\right)$ and 
$\phi=\mathcal{O}\left(\delta\left(t_{b} / \epsilon\right)^{1 / 2}\right)=\delta \epsilon^{-3 / 4} \ll 1$. From the kinematic condition, (3d), we then find that $w=\mathcal{O}\left(\epsilon^{3 / 4}\right)$. Hence, we introduce the following scales for this regime:

$$
\begin{aligned}
& t=\epsilon^{-1 / 2} t^{*}, h=\epsilon^{1 / 4} h^{*}, \quad z=\epsilon^{1 / 4} z^{*}, \\
& w=\epsilon^{3 / 4} w^{*}, \phi=\frac{\delta}{\epsilon^{3 / 4}} \phi^{*}
\end{aligned}
$$

and obtain the problem:

$$
\begin{gathered}
\partial_{t^{*}} \phi^{*}+w^{*} \partial_{z^{*}} \phi^{*}=\partial_{z^{*} z^{*}} \phi^{*}, \\
w^{*}=-\frac{h^{*} z^{* 2}}{2}+\frac{z^{* 3}}{6}, \\
z^{*}=h^{*}: \\
\partial_{z^{*}} \phi^{*}=-\frac{1}{\beta}, \\
\partial_{t^{*}} h^{*}-w^{*}=0 \\
z^{*}=0: \\
\frac{\partial \phi^{*}}{\partial z^{*}}=0, \\
t^{*} \rightarrow 0: \quad h^{*} \rightarrow\left(2 t^{*} / 3\right)^{-1 / 2}
\end{gathered}
$$

where the final conditions come from matching.

To examine when the solution to (14a)-(14e) may cease to be valid it is instructive to consider the long-time limit the problem. This can be easily seen by setting

$$
\begin{aligned}
& z^{*}=\frac{1}{\sqrt{2 / 3 t^{*}}} \xi, \quad h^{*}=\frac{1}{\sqrt{2 / 3 t^{*}}} f, \\
& \phi^{*}\left(z^{*}, t^{*}\right)=\psi\left(\xi, t^{*}\right),
\end{aligned}
$$

and obtain the problem

$$
\frac{3}{2} \frac{1}{t^{*}} \partial_{t^{*}} \psi+\frac{3}{4} \frac{1}{t^{* 2}} \xi\left(1-\frac{3}{2} f \xi+\frac{1}{2} \xi^{2}\right) \partial_{\xi} \psi=\partial_{\xi \xi} \psi
$$


at $\xi=f$

$$
\begin{aligned}
& \left(\frac{2}{3} t^{*}\right)^{1 / 2} \partial_{\xi} \psi=-\frac{1}{\beta} \\
& \frac{2}{3} t^{*} \partial_{t^{*}} f-\frac{1}{3} f+\frac{1}{3} f^{3}=0
\end{aligned}
$$

at $\xi=0$

$$
\partial_{\xi} \psi=0
$$

with the solution

$$
\begin{aligned}
f\left(t^{*}\right) & =\frac{1}{\sqrt{1+c_{1} / t^{*}}} \text { and } \\
\psi\left(\xi, t^{*}\right) & =-\frac{1}{\beta}\left(\frac{2}{3} t^{*}\right)^{3 / 2}+\left(-\frac{\sqrt{3}}{2 \sqrt{2}} \frac{1}{\beta} \xi^{2}+c_{2}\right) t^{*-1 / 2}+\cdots
\end{aligned}
$$

for $t^{*} \gg 1$. This solution is compared with the numerical solution in figure 2 .

The solution breaks down either when the evaporation becomes important for the evolution of the layer thickness $h$ or when the volume fraction $\phi$ becomes $\mathcal{O}(1)$. From (15) and (17), we obtain $\phi=\mathcal{O}\left(\delta t^{3 / 2}\right)$, and this becomes $\mathcal{O}(1)$ when $t=\mathcal{O}\left(\delta^{-2 / 3}\right)$. Similarly, we find $h=\mathcal{O}\left(t^{-1 / 2}\right)$ thus $h_{t}=\mathcal{O}\left(h^{3}\right)=\mathcal{O}\left(t^{-3 / 2}\right)$ and this will be of the same $\mathcal{O}(\delta)$ as the evaporation when $t=\mathcal{O}\left(\delta^{-2 / 3}\right)$. This too yields $t=\mathcal{O}\left(\delta^{-2 / 3}\right)$ for the breakdown of validity, marking the transition to a new time regime.

3. Long time scale $\left(t=\mathcal{O}\left(\delta^{-2 / 3}\right)\right)$

For this new time regime, we therefore scale

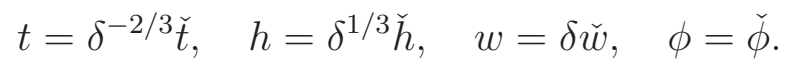




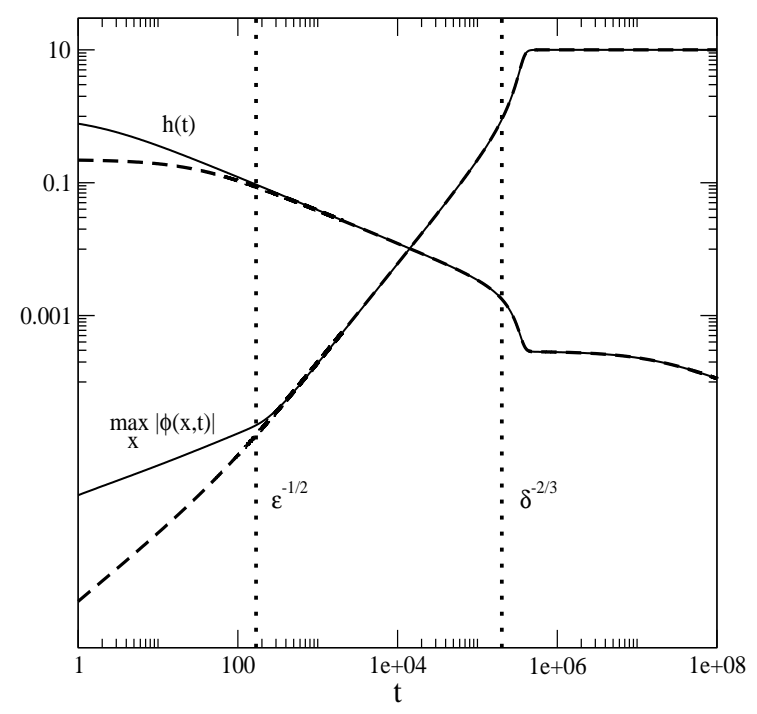

FIG. 3. Comparison of the numerical and asymptotic results in the small evaporation regime, for $\epsilon=3.5 \times 10^{-5}, \delta=1.1 \times 10^{-8}$, and for constant viscosity $\mu=0$. The solid curves denote the numerical results for $h(t)$ and $\max _{x}|\phi(x, t)|$ for (3). The dashed lines show the numerical for the leading order asymptotic problem in the late time regime (20). The two vertical dotted lines correspond to the times $t=\epsilon^{-1 / 2}=169$ and $t=\delta^{-2 / 3}=2.02 \times 10^{5}$, respectively.

Substitution into (3) yields

$$
\begin{aligned}
& \partial_{\check{t}} \check{\phi}+\check{w} \partial_{\check{z}} \check{\phi}=\frac{\epsilon}{\delta^{4 / 3}} \partial_{\check{z}}\left(e^{\check{\phi}} \partial_{\check{z}} \check{\phi}\right), \\
& \check{w}=\int_{0}^{\check{z}}(\check{z}-\check{q})(\check{h}-\check{q}) e^{\mu \check{\phi}} d \check{q}, \\
& \text { at } \check{z}=\check{h} \text { : } \\
& \frac{\epsilon}{\delta^{4 / 3}} e^{\check{\phi}} \partial_{\check{z}} \check{\phi}=-\frac{1}{\beta}(1+\gamma \check{\phi})(1-\beta \check{\phi}), \\
& \partial_{\check{t}} \check{h}-\check{w}=-(1+\gamma \check{\phi}) \text {, } \\
& \text { at } \check{z}=\check{h} \text { : } \\
& \partial_{\check{z}} \check{\phi}=0 \text {. }
\end{aligned}
$$

We let

$$
\begin{aligned}
& \check{\phi}=\check{\phi}_{0}+\frac{\delta^{4 / 3}}{\epsilon} \check{\phi}_{1}+\cdots, \\
& \check{h}=\check{h}_{0}+\frac{\delta^{4 / 3}}{\epsilon} \check{h}_{1}+\cdots, \\
& \check{w}=\check{w}_{0}+\frac{\delta^{4 / 3}}{\epsilon} \check{w}_{1}+\cdots,
\end{aligned}
$$


and obtain that $\check{\phi}_{0}$ depends only on $t$. Hence at next order the problem is

$$
\begin{aligned}
\frac{d \check{\phi}_{0}}{d \check{t}} & =-\frac{1}{\beta} \frac{\left(1+\gamma \check{\phi}_{0}\right)\left(1-\beta \check{\phi}_{0}\right)}{\check{h}_{0}} \\
\frac{d \check{h}_{0}}{d \check{t}} & =-\frac{1}{3} e^{\mu \check{\phi}_{0}} \check{h}_{0}^{3}-\left(1+\gamma \check{\phi}_{0}\right) \\
\check{w}_{0} & =e^{\mu \check{\phi}_{0}(t)}\left(-\frac{\check{h}_{0} \check{z}^{2}}{2}+\frac{\check{z}^{3}}{6}\right) .
\end{aligned}
$$

The initial conditions for this system comes from matching back with the medium time layer,

$$
\phi_{0} \rightarrow 0, \quad h_{0} \sim(2 t / 3)^{-1 / 2} \quad \text { for } t \rightarrow 0 .
$$

The solution to this problem is obtained numerically and shown in figure 3 , where it is compared to the solution for the full lubrication model (3). As $t \rightarrow \infty$, the solution for $h_{0}$ and $\phi_{0}$ tends monotonically to the equilibrium $h_{0}=0, \phi_{0}=-1 / \gamma$ of the ODE system (20a), (20b). The solutions are in excellent agreement even in the later stages of the medium time regime and throughout the late time regime. This is true if the diffusion remains strong enough to keep the volume fraction profile constant throughout the film, even as the exponential term on the right hand side of (18a) becomes smaller as $\phi_{0}$ approaches $-1 / \gamma$. This is the case if the condition $\exp (1 / \gamma) \ll \epsilon / \delta^{4 / 3}$ (or equivalently the very small evaporation limit $\left.\delta \ll \exp (-3 /(4 \gamma)) \epsilon^{3 / 4}\right)$ is satisfied, which imposes a lower bound for $\gamma$ in order that no skin forms. For the values for $\epsilon$ and $\delta$ chosen in figure 3 , this bound is about $\gamma=1 / \ln \left(\epsilon / \delta^{4 / 3}\right)=0.07$. Note our choice of $\gamma$ is larger and indeed we found that the numerical solutions of (3) have flat volume fraction profiles. We have tested this bound by carrying out further simulations of (3) with smaller values of $\gamma$ but all other parameters unchanged. Already for $\gamma=0.05$, the volume fraction profiles varied significantly across the film in the time period where $\phi$ increased rapidly towards the equilibrium value, and this effect became more pronounced for smaller $\gamma$; for $\gamma=0.035$, the variation was more than $50 \%$. We conclude that our estimate of the bound that defines very small evaporation is reasonably accurate.

\section{The effect of volume fraction dependent viscosity}

So far, all the figures only show results for the case where $\mu=0$, i.e., the viscosity does not depend on the volume fraction of the solvent. Indeed, setting $\mu=1$ hardly changes the 


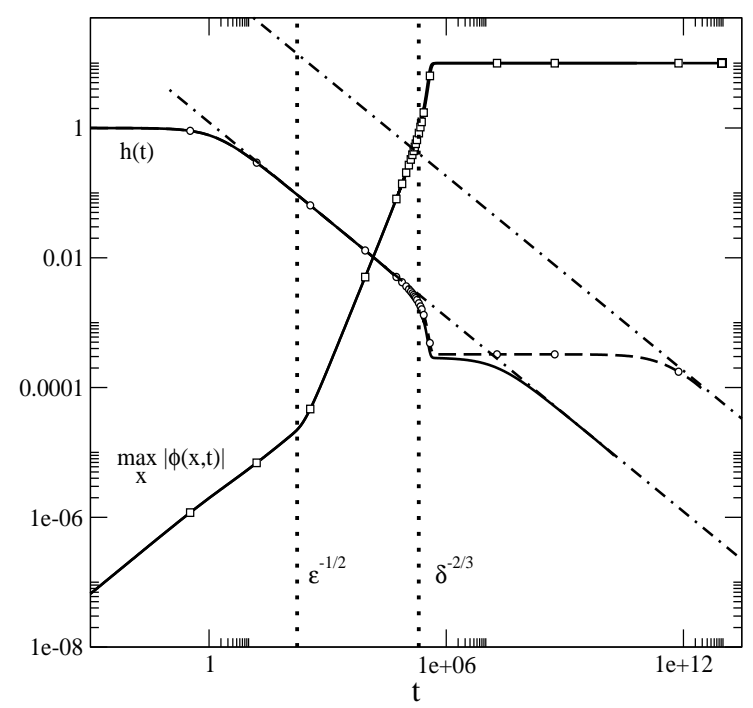

FIG. 4. Comparison of the numerical solution of (3) for $\mu=0$ (solid lines) with the results for $\mu=1$ (dashed lines with symbols). The dot-dashed lines indicate the long time behaviour for $h$ according to (20), which is $h \sim(2 / 3 t)^{-1 / 2}$ for $\mu=0$ (bottom line) and $h \sim(2 \exp (-1 / \gamma) / 3 t)^{-1 / 2}$ for $\mu=1$ (top line).

evolution the film thickness and the maximum value of $\phi(x, t)$, with one notable exception. Once a significant amount of solvent has evaporated throughout the film (i.e. $\phi$ approaches $-1 / \gamma$ everywhere rather than only in a thin boundary layer) the thinning due to centrifugal forces slows down dramatically as the viscosity in the bulk increases significantly. In fact, when we compare the evolution of $h(t)$ for the two choices of $\mu$ in fig. 4 , the two lines only disagree after $t=\mathcal{O}\left(\delta^{-2 / 3}\right)$, where the effect of evaporation on the evolution of $h$ becomes significant. For $\mu=0$, thinning returns to $h \sim(2 t / 3)^{-1 / 2}$ after a while; for $\mu=1$ the thinning is slower and eventually becomes $h \sim(2 \exp (-1 / \gamma) / 3 t)^{-1 / 2}$. This behaviour follows from (20)(b) since $\breve{\phi}_{0} \rightarrow-1 / \gamma$ as $\breve{t} \rightarrow \infty$.

Note that for $\max _{x}|\phi(x, t)|$, the results for the two values of $\mu$ are indistinguishable in the figure. Also note that for the other cases, i.e. medium and large evaporation, the effect of replacing $\mu=0$ by $\mu=1$ is qualitatively the same for the full model, in the sense that in the numerical solution $\max _{x}|\phi(x, t)|$ is largely unaffected and only the final long time behaviour of $h(t)$ changes from $h \sim(2 / 3 t)^{-1 / 2}$ to $h \sim(2 \exp (-1 / \gamma) / 3 t)^{-1 / 2}$.

Physically having the viscosity change only manifests itself when the concentration of solvent changes sufficiently from its initial value to significantly alter the viscosity. For large 
and medium evaporation rates this only occurs in a thin surface skin layer. In this layer the shear stress is very small, due to the proximity of the surface and so the viscosity changes have no appreciable effect on the behaviour. For small and very small evaporation rates the spatial uniformity of the concentration implies that the viscosity changes simply slow the thinning down and alter the velocities in a uniform manner. We do note that the increased viscosity may result in elongation stresses becoming significant in the layer however for the one dimensional model studied here we have neglected such effects. For these reasons in the analysis of the remaining cases in this paper, we focus exclusively on $\mu=0$.

B. Medium evaporation $\left(\epsilon^{3 / 4} \ll \delta \ll \epsilon^{1 / 2} \ll 1\right)$

\section{Early time scale $(t=\mathcal{O}(1))$}

For $t=\mathcal{O}(1)$, we obtain the same results as for the early time regime in small evaporation rate. In particular we note that the analysis of the boundary layer is valid provided $\delta \ll \epsilon^{1 / 2}$. However, the transition to the next time regime occurs via the third scenario $c$ ) listed in section IV A 2 , since for $\delta \gg \epsilon^{3 / 4}$, the time scale $t_{c}=\mathcal{O}\left(\epsilon / \delta^{2}\right)$ is the shortest of the three.

\section{Medium time scale $\left(t=\mathcal{O}\left(\epsilon / \delta^{2}\right)\right)$}

Using the earlier results we know that there is a boundary layer in which the concentration is expected to start to alter significantly. Hence in the outer layer we take these scalings

$$
\begin{aligned}
t & =\frac{\epsilon}{\delta^{2}} \breve{t}, \quad h=\frac{\delta}{\epsilon^{1 / 2}} \breve{h}, \quad z=\frac{\delta}{\epsilon^{1 / 2}} \breve{z}, \\
\phi & =\breve{\phi} \quad, \quad w=\left(\frac{\delta}{\epsilon^{1 / 2}}\right)^{3} \breve{w} .
\end{aligned}
$$

This yields

$$
\begin{aligned}
\partial_{\breve{t}} \breve{\phi}+\breve{w} \partial_{\breve{z}} \breve{\phi} & =\frac{\epsilon^{3}}{\delta^{4}} \partial_{\breve{z}}\left(e^{\breve{\phi}} \partial_{\breve{z}} \breve{\phi}\right), \\
\breve{w} & =-\frac{\breve{h} \breve{z}^{2}}{2}+\frac{\breve{z}^{3}}{6},
\end{aligned}
$$


at $\breve{z}=\breve{h}$ :

$$
\begin{aligned}
\frac{\epsilon^{3 / 2}}{\delta^{2}} e^{\breve{\phi}} \partial_{\breve{z}} \breve{\phi} & =-\frac{1}{\beta}(1+\gamma \breve{\phi})(1-\beta \breve{\phi}), \\
\partial_{\breve{t}} \breve{h}-\breve{w} & =-\frac{\epsilon^{3 / 2}}{\delta^{2}}(1+\gamma \breve{\phi}),
\end{aligned}
$$

at $\breve{z}=0$ :

$$
\partial_{\breve{z}} \breve{\phi}=0
$$

as $\breve{t} \rightarrow 0$ :

$$
\breve{\phi} \rightarrow 0, \quad \breve{h} \rightarrow(2 \breve{t} / 3)^{-1 / 2}
$$

Because of the regime we are considering we know that $\epsilon^{3 / 2} / \delta^{2} \ll 1$, thus to leading order we obtain an outer problem whose solution can readily be found to be

$$
\breve{\phi}=0, \quad \breve{h}=\left(\frac{2}{3} \breve{t}\right)^{-1 / 2}
$$

In the boundary layer we introduce the scalings

$$
\breve{z}=\breve{h}+\frac{\epsilon^{3 / 2}}{\delta^{2}} \overline{\bar{z}}, \quad \breve{\phi}=\overline{\bar{\phi}}, \quad \breve{t}=\overline{\bar{t}}
$$

This leads to the leading order inner problem

$$
\partial_{\overline{\bar{t}}} \overline{\bar{\phi}}+\left(1+\gamma \overline{\bar{\phi}}(0)-\frac{3 \overline{\bar{z}}}{4 \overline{\bar{t}}}\right) \partial_{\overline{\bar{z}}} \overline{\bar{\phi}}=\partial_{\overline{\bar{z}}}\left(e^{\overline{\bar{\phi}}} \partial_{\overline{\bar{z}}} \overline{\bar{\phi}}\right),
$$

at $\overline{\bar{z}}=0$ :

$$
e^{\overline{\bar{\phi}}} \partial_{\overline{\bar{z}}}^{\overline{\bar{\phi}}}=-\frac{1}{\beta}(1+\gamma \overline{\bar{\phi}})(1-\beta \overline{\bar{\phi}}),
$$

as $\overline{\bar{z}} \rightarrow-\infty$ :

$$
\overline{\bar{\phi}} \rightarrow 0
$$

as $\overline{\bar{t}} \rightarrow 0$ :

$$
\overline{\bar{\phi}} \rightarrow 0 \text {. }
$$


As time increases the analysis breaks down when either the boundary layer thickness equals the thickness $h$ of the film or evaporation begins to significantly modify the evolution of $h$. In fact, we expect that the time scale for both events must be the same: If $\phi$ is order one in the boundary layer, then evaporation has a leading order effect on the film thickness exactly when the boundary layer thickness is no longer small compared to $h$ itself. From a diffusion balance in (25a), we find that the boundary layer grows like $\epsilon^{1 / 2} t^{1 / 2}$; from (23), we obtain $h=\mathcal{O}\left(t^{-1 / 2}\right)$. Equating the two yields $t=\mathcal{O}\left(\epsilon^{-1 / 2}\right)$. On this time scale $h_{t}=\mathcal{O}\left(\epsilon^{3 / 4}\right)$; this does not seem to balance evaporation i.e. the right hand side of (3d), which appears to be $\mathcal{O}(\delta) \gg \epsilon^{3 / 4}$. However, we have to take into account that $\phi(h)$ is already very close to $-1 / \gamma$ so that $(1+\gamma \phi(h))=\mathcal{O}\left(\epsilon^{3 / 4} / \delta\right)$.

\section{Long time scale $\left(t=\mathcal{O}\left(\epsilon^{-1 / 2}\right)\right)$}

In this time regime, we scale according to

$$
\begin{aligned}
t & =\epsilon^{-1 / 2} \tilde{t}, & h & =\epsilon^{1 / 4} \tilde{h}, \quad z=\epsilon^{1 / 4} \tilde{z}, \\
\phi & =\tilde{\phi}, \quad & w & =\epsilon^{3 / 4} \tilde{w} .
\end{aligned}
$$

Note that we do not have a boundary layer i.e. there is only one spatial scaling. Introducing these scalings yields

$$
\begin{aligned}
\partial_{\tilde{t}} \tilde{\phi}+\tilde{w} \partial_{\tilde{z}} \tilde{\phi} & =\partial_{\tilde{z}}\left(e^{\tilde{\phi}} \partial_{\tilde{z}} \tilde{\phi}\right), \\
\tilde{w} & =-\frac{\tilde{h} \tilde{z}^{2}}{2}+\frac{\tilde{z}^{3}}{6},
\end{aligned}
$$

at $\tilde{z}=\tilde{h}$ :

$$
\begin{aligned}
\frac{\epsilon^{3 / 4}}{\delta} e^{\tilde{\phi}} \partial_{\tilde{z}} \tilde{\phi} & =-\frac{1}{\beta}(1+\gamma \tilde{\phi})(1-\beta \tilde{\phi}), \\
\frac{\epsilon^{3 / 4}}{\delta}\left(\partial_{\tilde{t}} \tilde{h}-\tilde{w}\right) & =-(1+\gamma \tilde{\phi}),
\end{aligned}
$$

at $\tilde{z}=0$ :

$$
\partial_{\tilde{z}} \tilde{\phi}=0,
$$

as $\tilde{t} \rightarrow 0$ :

$$
\tilde{\phi} \rightarrow 0, \quad \tilde{h} \rightarrow(2 \tilde{t} / 3)^{-1 / 2}
$$




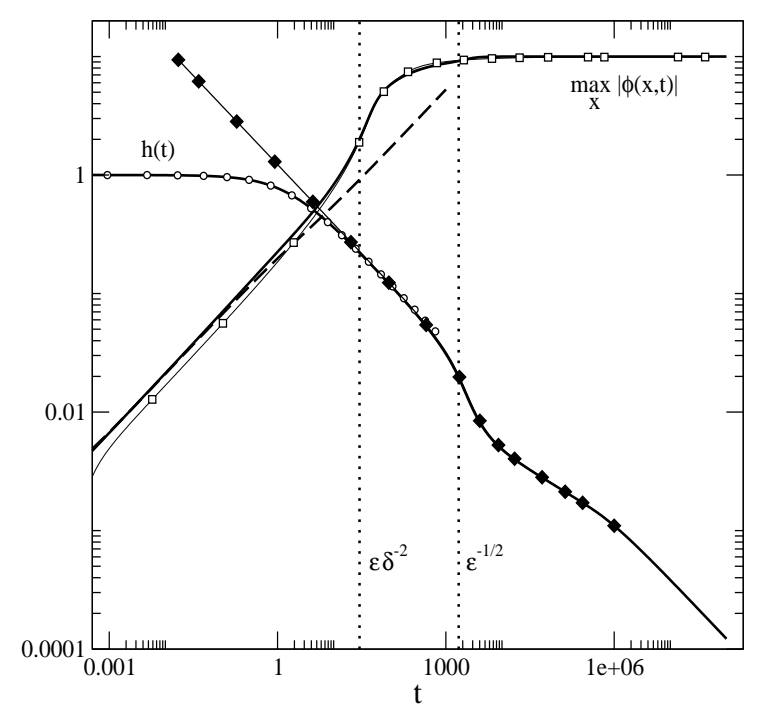

FIG. 5. Comparison of the numerical and asymptotic results in the medium evaporation regime, for $\epsilon=3.5 \times 10^{-7}, \delta=1.1 \times 10^{-4}$, and for constant viscosity $\mu=0$. The thick solid curves denote the numerical solutions of (3). The circles and the dashed line denote the results for the asymptotic approximations in the early time regime (7) and (9) (circles for $h(t)$ and the dashed line for $\left.\max _{x}|\phi(x, t)|\right)$. The solution for $h(t)$ remains valid to leading order in the medium time regime (see (23)), while the solution of the leading order inner problem (25) for $\max _{x}|\phi(x, t)|$ in this time regime is given by squares; it continues to agree well with the solution of (3) also in the long-time regime. The thin line with the solid diamonds corresponds to the solution for $h(t)$ to the leading order asymptotic problem (29), (31) in the long time regime. The two vertical dotted lines indicate the times $t=\epsilon / \delta^{2}=28.9$ and $t=\epsilon^{-1 / 2}=1.69 \times 10^{3}$, respectively.

We let

$$
\begin{aligned}
\tilde{\phi} & =\tilde{\phi}_{0}+\frac{\epsilon^{3 / 4}}{\delta} \tilde{\phi}_{1}+\cdots, \\
\tilde{h} & =\tilde{h}_{0}+\frac{\epsilon^{3 / 4}}{\delta} \tilde{h}_{1}+\cdots, \\
\tilde{w} & =\tilde{w}_{0}+\frac{\epsilon^{3 / 4}}{\delta} \tilde{w}_{1}+\cdots,
\end{aligned}
$$

and obtain to leading order

$$
\begin{aligned}
\partial_{\tilde{t}} \tilde{\phi}_{0}+\tilde{w}_{0} \partial_{\tilde{z}} \tilde{\phi}_{0} & =\partial_{\tilde{z}}\left(e^{\tilde{\phi}_{0}} \partial_{\tilde{z}} \tilde{\phi}_{0}\right), \\
\tilde{w}_{0} & =-\frac{\tilde{h}_{0} \tilde{z}^{2}}{2}+\frac{\tilde{z}^{3}}{6},
\end{aligned}
$$



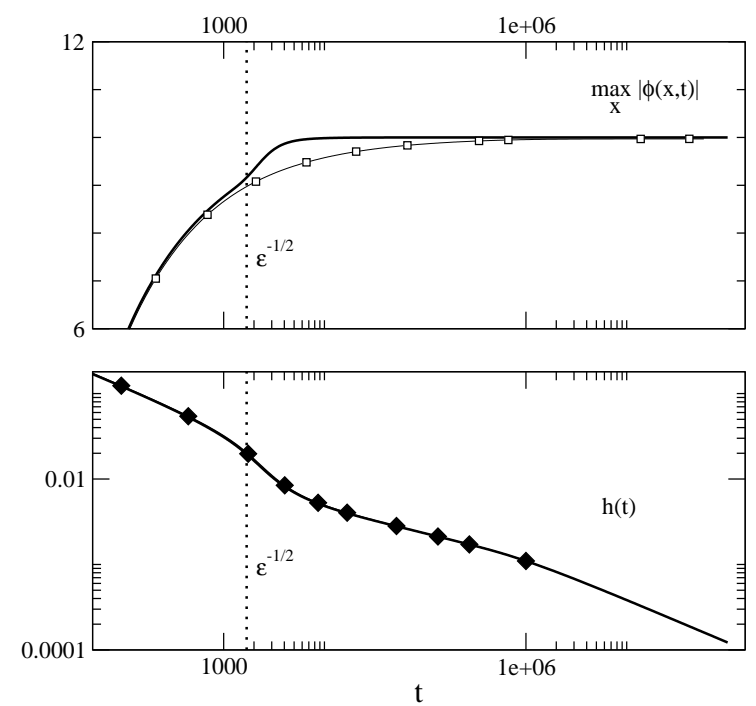

FIG. 6. Expanded details of the graphs given in fig. 5 for $\max _{x}|\phi(x, t)|$ and $h(t)$. All parameters, line styles and symbols identical to fig. 5 .

at $\tilde{z}=\tilde{h}_{0}$ :

$$
\tilde{\phi}_{0}=-\frac{1}{\gamma}
$$

at $\tilde{z}=0$ :

$$
\partial_{\tilde{z}} \tilde{\phi}_{0}=0
$$

as $\tilde{t} \rightarrow 0$ :

$$
\tilde{\phi}_{0} \rightarrow 0, \quad \tilde{h}_{0} \rightarrow(2 \tilde{t} / 3)^{-1 / 2}
$$

Note that the two boundary conditions at $z=\tilde{h},(27 \mathrm{c})$ and $(27 \mathrm{~d})$, result in a single leading order condition at $z=\tilde{h}_{0},(29 \mathrm{c})$. To close the problem, we need an additional condition, which comes from carrying out the expansion for (27c) and (27d) to next order,

$$
\begin{aligned}
e^{\tilde{\phi}_{0}} \partial_{\tilde{z}} \tilde{\phi}_{0} & =-\frac{\gamma}{\beta}\left(\gamma \tilde{\phi}_{0 z}+\gamma \tilde{\phi}_{1}\right)\left(1-\beta \tilde{\phi}_{0}\right), \\
\partial_{\tilde{t}} \tilde{h}_{0}-\tilde{w}_{0} & =-\left(\gamma \tilde{\phi}_{0 z}+\gamma \tilde{\phi}_{1}\right),
\end{aligned}
$$

at $z=\tilde{h}_{0}$, from which we obtain by elimination of $\tilde{\phi}_{1}\left(\tilde{h}_{0}\right)$ the following condition at $z=\tilde{h}_{0}$,

$$
\partial_{\hat{t}} \tilde{h}_{0}-\tilde{w}_{0}=\frac{\beta \gamma}{\beta+\gamma} e^{-1 / \gamma} \partial_{\tilde{z}} \tilde{\phi}_{0}
$$




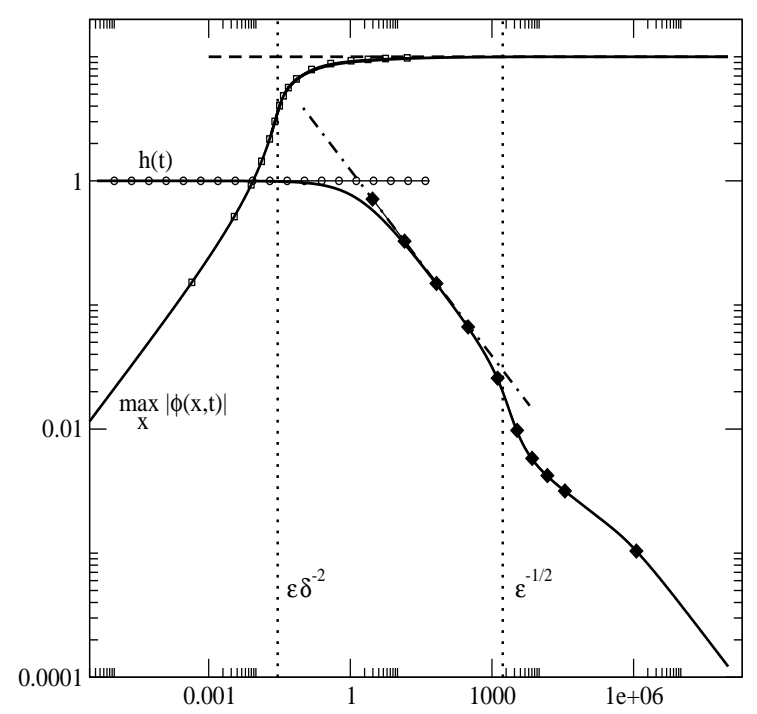

FIG. 7. Comparison of the numerical and asymptotic results in the large evaporation regime, for $\epsilon=3.5 \times 10^{-7}, \delta=3.48 \times 10^{-3}$, and for constant viscosity $\mu=0$. The solid curves denote the numerical solutions of (3). The thin lines with circles and the squares denote the results for the asymptotic problems in the early time regime (circles for $h(t)$, which is constant to one, and squares for $\max _{x}|\phi(x, t)|$, obtained from (34)). In the medium time regime, the solution for the asymptotic problems (35), (40) is given by a dash-dotted line for $h(t)$ and by a dashed line for $\max _{x}|\phi(x, t)|=1 / \gamma$. In the long time regime, the solution to (29), (31) is indicated by a thin line with solid diamonds for $h(t)$. The two vertical dotted lines correspond to the times $t=\epsilon / \delta^{2}=2.89 \times 10^{-2}$ and $t=\epsilon^{-1 / 2}=1.69 \times 10^{3}$, respectively.

The solution of the leading order problem (29) is shown in figure 5 (dashed-dotted line for $\tilde{h}_{0}$ and square symbols for $\tilde{\phi}_{0}$ ). In order to capture the small bump shown in the figure 5 (top) for $\max _{x}|\phi|$, higher order corrections to the the leading order asymptotic results are required. In practice, the solution to (29) will change on a very long time scale (long even in terms of $\tilde{t}$ ) due to the small values of $\gamma$. This is an interesting problem, which we shall not pursue further here. 


\section{Large evaporation $\left(\epsilon^{1 / 2} \ll \delta \ll 1\right)$}

\section{1. $\quad$ Early time scale $\left(t=\mathcal{O}\left(\epsilon / \delta^{2}\right)\right)$}

We anticipate a thin boundary layer will be created by the evaporation at the surface. Appropriate variables in the boundary layers are

$$
z=h(t)+\frac{\epsilon}{\delta} \hat{z} \quad \text { and } \quad \phi(z, t)=\hat{\phi}(\hat{z}, t) .
$$

This scaling allows for order one values of $\phi$ in the boundary layer and balances the terms on the left and right hand side of $(3 \mathrm{c})$. Balancing the time derivative in the bulk with the diffusion requires that we rescale time by

$$
t=\frac{\epsilon}{\delta^{2}} \hat{t}
$$

which implies a short time regime (since $\left.\epsilon / \delta^{2} \ll 1\right)$. With these scalings, the leading order boundary layer problem becomes

$$
\text { at } \hat{z}=0 \begin{aligned}
& \partial_{\hat{t}} \hat{\phi}+(1+\gamma \hat{\phi}(0)) \partial_{\hat{z}} \hat{\phi}=\partial_{\hat{z}}\left(e^{\hat{\phi}} \partial_{\hat{z}} \hat{\phi}\right), \\
& e^{\hat{\phi}} \partial_{\hat{z}} \hat{\phi}=-\frac{1}{\beta}(1+\gamma \hat{\phi})(1-\beta \hat{\phi}),
\end{aligned}
$$

A far-field condition at $z \rightarrow-\infty$ comes from matching to the outer problem. On the short time scale (33), the leading order outer problem becomes trivial; it has the constant solution $h=1$ and $\phi=0$. Matching yields

$$
\hat{\phi} \rightarrow 0 \quad \text { as } \quad \hat{z} \rightarrow-\infty
$$

The solutions to the leading order asymptotic problems are compared to the solutions of the full model are compared in fig. 7. The figure also contains lines for the solutions to the asymptotic problems in the medium and long time regime stated in the next two sections.

On the early time scale $\partial_{t} h=\mathcal{O}\left(\delta^{2} / \epsilon\right) \gg w=\mathcal{O}(1)$ in equation (3d). This regime ends once $t=\mathcal{O}(1)$ when $\partial_{t} h$ and $w$ balance.

\section{Medium time scale $(t=\mathcal{O}(1))$}

Since also $h=\mathcal{O}(1)$ also $w=\mathcal{O}(1)$ for $z=\mathcal{O}(1)$, we leave all variables in their original scaling in this regime. Hence the leading order problem is directly obtained from problem 
(3), and has the solution

$$
\phi_{0}=0, \quad h_{0}=\frac{1}{\sqrt{1+2 t / 3}} .
$$

However, this solution does not satisfy the boundary condition $(3 \mathrm{c})$, so we need to introduce a boundary layer at $z=h(t)$.

a. Boundary layer problem Setting

$$
z=h(t)+\epsilon^{1 / 2} z^{*}
$$

the boundary problem reads

$$
\begin{array}{r}
\partial_{t} \phi+\left(\frac{\delta}{\epsilon^{1 / 2}}(1+\gamma \phi(0))-\frac{h^{2} z^{*}}{2}+\epsilon \frac{z^{* 3}}{6}\right) \partial_{z^{*}} \phi= \\
\partial_{z^{*}}\left(e^{\phi} \partial_{z^{*}} \phi\right),
\end{array}
$$

at $z^{*}=0$ :

$$
\frac{\epsilon^{1 / 2}}{\delta} e^{\phi} \partial_{z^{*}} \phi=-\frac{1}{\beta}(1+\gamma \phi)(1-\beta \phi),
$$

as $z^{*} \rightarrow-\infty$ :

$$
\tilde{\phi} \rightarrow 0
$$

We consider the expansions

$$
\phi=\phi_{0}+\frac{\epsilon^{1 / 2}}{\delta} \phi_{1}+O\left(\epsilon / \delta^{2}\right), \quad h=h_{0}+\frac{\epsilon^{1 / 2}}{\delta} h_{1}+O\left(\epsilon / \delta^{2}\right)
$$

and obtain

$$
\phi_{0}(0)=-\frac{1}{\gamma}, \quad \phi_{1}(0)=\frac{\beta \gamma}{\beta+\gamma} e^{-1 / \gamma} \partial_{z^{*}} \phi_{0}(0),
$$

where the leading order problem is

$$
\begin{aligned}
\partial_{t} \phi_{0} & +\left(-\frac{\beta \gamma e^{-1 / \gamma}}{\beta+\gamma} \partial_{z^{*}} \phi_{0}(0)-\frac{h_{0}^{2} z^{*}}{2}\right) \partial_{z^{*}} \phi_{0} \\
& =\partial_{z^{*}}\left(e^{\phi} \partial_{z^{*}} \phi\right), \\
\phi_{0}(0) & =-\frac{1}{\gamma},
\end{aligned}
$$


and as $z^{*} \rightarrow-\infty$ :

$$
\phi_{0} \rightarrow 0
$$

Also, $\phi_{0} \rightarrow 0$ as $t \rightarrow 0$.

We note that as $t \rightarrow \infty$ then $h_{0}=\mathcal{O}\left(t^{-1 / 2}\right)$. Since the diffusion balance yields $z^{*}=\mathcal{O}\left(t^{1 / 2}\right)$ and the boundary layer grows to the size of the film thickness $\mathcal{O}\left(\epsilon^{1 / 2} t^{1 / 2}\right)=\mathcal{O}\left(t^{-1 / 2}\right)$ which suggests that the next time regime is $t=\mathcal{O}\left(\epsilon^{-1 / 2}\right)$.

\section{Long time scale $\left(t=\mathcal{O}\left(\epsilon^{-1 / 2}\right)\right)$}

For the long-time behaviour we obtain the same scales as in (26) in section (IV B 3). This results in the same set of equations as in that section.

\section{CONCLUSIONS}

Our analysis of spin coating a polymer blended in a volatile solvent shows that in the high Peclet number regime, there are essentially three asymptotic regimes that describe distinct paths of the film thinning process starting from the initial liquid layer to the final solid film and can be described by corresponding asymptotic boundary value problems for the small, medium and large evaporation limits. They are distinguished by the relationship between three main parameters, $\epsilon$ the ratio of diffusion to advection, $\delta$ the ratio of evaporation to advection and $\exp (-\gamma)$ the ratio of the diffusivity of the initial mixture and the pure polymer.

We show that, while the basic mechanisms discussed in detail by Bornside et al. $^{2}$ are valid, the important practical problem of understanding how to prevent the eventual skin formation can in fact be quantified. We predict that for the very small evaporation limit, when $\delta \ll \exp (-3 /(4 \gamma)) \epsilon^{3 / 4}$ is satisfied, no skin formation will occur.

In the remaining small, medium and large evaporation cases, where there is always skin formation, we show that the time scales at which the skin appears and the details of its formation are different. In the small evaporation regime, the solvent is initially depleted in a thin boundary layer region near the liquid surface. However, the boundary layer spreads out until it spans the entire film, after which the volume fraction profile flattens out across 
the film. If the evaporation is very small, this flat profile is maintained until all solvent is evaporated. But if it is not very small, the changes in diffusivity can give rise to steeper volume fraction gradients and eventually to skin formation.

In the medium evaporation regime, an order one change of the volume fraction occurs within the surface boundary layer, giving rise to a skin within a medium time scale. Underneath the skin, the polymer concentration is still at its initial value. After the skin has formed, depletion due to evaporation is slowed down because diffusion of the solvent through the boundary layer is greatly diminished. However, there are still significant volume fraction gradients so the volume profile continues to evolve, albeit on a very slow time scale. These gradients are driven by the fact that the material at surface is almost pure polymer.

The large evaporation regime is qualitatively similar to the medium evaporation case, except that the skin arises on a very fast timescale, i.e. much smaller than order one, before any liquid has been ejected due to the centrifugal forces.

The behaviour described above is characteristic for liquids of constant viscosity as well as for concentration dependent viscosity. In fact our numerical results are almost indistinguishable during the time regime when evaporation is still dominant. A dramatic quantitative change sets in after significant amounts of the solvent has evaporated throughout the film and the thinning for the liquid with concentration dependent viscosity slows down considerably.

For practical purposes, we note that for a given material the parameter $\epsilon$ can be modified by a reasonable amount by changing the spinning speed of the disk or modified dramatically by the initial volume fraction of the solvent, while $\delta$ can be modified reasonably either by changing the spinning speed and changing the overlying solvent volume fraction, whereas $\exp (-\gamma)$ depends very sensitively on both the initial volume fraction of the solvent and the overlying solvent volume fraction.

There will be further aspects to consider in the future that have not been explored in detail previously in the literature or in this paper. Initially they will concern the possible formation of instabilities of the flow in higher dimensions. Extending the number of constituents of the polymer blends will introduce new phenomena such as phase separation or liquid-liquid dewetting. This is the subject of later investigations. 


\section{ACKNOWLEDGEMENT}

The authors gratefully acknowledge the generous support of OCCAM (Oxford Centre for Collaborative Applied Mathematics) under support supplied by Award No. KUK-C1013-04, made by the King Abdullah University of Science and Technology (KAUST). CPP and BW are especially grateful for the support and hospitality during their OCCAM Visiting Fellowships. The authors also enjoyed lively and very fruitful discussions with John R. Ockendon and Chris J.W. Breward.

\section{REFERENCES}

${ }^{1}$ A. Acrivos, M. J. Shah, and E. E. Petersen. On the flow of a non-newtonian liquid on a rotating disk. J. Appl. Phys., 31:963-968, 1960.

${ }^{2}$ D. Bornside, C. Macocsko, and L. Scriven. Spin coating: One-dimensional model. J. Appl. Phys., 66:5185-5193, 1989.

${ }^{3}$ C.-C. Changa, C.-L. Paia, W.-C. Chena, and S. A. Jenekheb. Spin coating of conjugated polymers for electronic and optoelectronic applications. Thin Solid Films, 479:254-260, 2005.

${ }^{4}$ P. de Gennes. Solvent evaporation of spin cast films: crust effects. Eur. Phys. J. E, 7:31-34, 2002.

${ }^{5}$ A. G. Emslie, F. T. Bonner, and L. G. Peck. Flow of a viscous liquid on a rotating disk. J. Appl. Phys., 29:858-862, 1958.

${ }^{6}$ D. P. B. III and M. Manley. Combined flow and evaporation of fluid on a spinning disk. Phys. Fluids, 9:870-875, 1997.

${ }^{7}$ P. Jukes, S. Heriot, J. Sharp, and R. Jones. Time-resolved light scattering studies of phase separation in thin film semiconducting polymer blends during spin-coating. Macromolecules, 38:2030-2032, 2005.

${ }^{8}$ J.-S. Kim, P. K. H. Ho, C. E. Murphy, and R. H. Friend. Phase separation in polyfluorenebased conjugated polymer blends: Lateral and vertical analysis of blend spin-cast thin films. Macromolecules, 37:2861-2871, 2004.

${ }^{9}$ F. Kreith, J. H. Taylor, and J. P. Chong. Heat and mass transfer from a rotating disk. J. Heat Transfer, 81:95-105, 1959. 
${ }^{10}$ C. J. Lawrence. The mechanics of spin coating of polymer films. Phys. Fluids, 31:27862795,1988 .

${ }^{11}$ C. J. Lawrence. Spin coating with slow evaporation. Physics of Fluids A-Fluid Dynamics, 2:453-456, 1990.

${ }^{12}$ A. Meyerhofer. Characteristics of resist films produced by spinning. J. Appl. Phys., 49:3993-3997, 1978.

${ }^{13}$ T. Okuzono, K. Ozawa, and M. Doi. Simple model of skin formation caused by solvent evaporation in polymer solutions. Phys. Rev. Lett., 97:136103, 2006.

${ }^{14}$ T. J. Rehg and B. G. Higgins. Spin coating of colloidal suspensions. AIChE Journal, 38:489-501, 1992.

${ }^{15}$ B. Reisfeld, S. G. Bankoff, and S. H. Davis. The dynamics and stability of thin liquid films during spin coating. i. films with constant rates of evaporation or absorption. J. Appl. Phys., 70:5258-5266, 1991.

${ }^{16}$ B. Reisfeld, S. G. Bankoff, and S. H. Davis. The dynamics and stability of thin liquid films during spin coating. ii. films with unit-order and large peclet numbers. J. Appl. Phys., 70:5267-5277, 1991.

${ }^{17}$ P. C. Sukanek. Spin coating. Journal of Imaging Technology, 11:184-190, 1985. 



\section{RECENT REPORTS}

36/10 Sequential Inverse Problems Bayesian Principles and the Logistic Duan Map Example

Farmer

Moroz

37/10 Circumferential buckling instability of a growing cylindrical tube

Moulton

Goriely

38/10 Preconditioners for state constrained optimal control problems with Moreau-Yosida penalty function

Stoll

Wathen

39/10 Local synaptic signaling enhances the stochastic transport of motor-driven cargo in neurons

Newby

Bressloff

40/10 Convection and Heat Transfer in Layered Sloping Warm-Water Aquifer

McKibbin

Hale

Style

Walters

41/10 Optimal Error Estimates of a Mixed Finite Element Method for

Goswami Parabolic Integro-Differential Equations with Non Smooth Initial Data

Pani

Yadav

42/10 On the Linear Stability of the Fifth-Order WENO Discretization

Motamed

Macdonald

Ruuth

43/10 Four Bugs on a Rectangle

Chapman

Lottes

Trefethen

44/10 Mud peeling and horizontal crack formation in drying clay

Style

Peppin

Cocks

45/10 Binocular Rivalry in a Competitive Neural Network with Synaptic Depression

Kilpatrick

Bressloff

46/10 A theory for the alignment of cortical feature maps during development

Bressloff

Oster

47/10 All-at-Once Solution if Time-Dependent PDE-Constrained Optimisation Problmes

Stoll

Wathen

48/10 Possible role of differential growth in airway wall remodeling in asthma

Moulton

Goriely

49/10 Variational Data Assimilation Using Targetted Random Walks

Cotter

Dashti

Robinson

Stuart

50/10 A model for the anisotropic response of fibrous soft tissues using

Flynn six discrete fibre bundles

Rubin

Nielsen 
51/10 STOCHSIMGPU Parallel stochastic simulation for the Systems Biology Toolbox 2 for MATLAB

Klingbeil

Erban

Giles

Maini

$52 / 10$ Order parameters in the Landau-de Gennes theory - the static

Majumdar and dynamic scenarios

53/10 Liquid Crystal Theory and Modelling Discussion Meeting

Majumdar

Mottram

54/10 Modeling the growth of multicellular cancer spheroids in a bioengi-

Loessner neered 3D microenvironment and their treatment with an anticancer drug

Flegg

Byrne

Hall

Moroney

Clements

Hutmacher

McElwain

55/10 Scalar Z, ZK, KZK, and KP equations for shear waves in incompressible solids

Destrade

Goriely

Saccomandi

56/10 The Influence of Bioreactor Geometry and the Mechanical Environment on Engineered Tissues

Osborne

ODea

Whiteley

Byrne

Waters

$57 / 10$ A numerical guide to the solution of the bidomain equations of

Pathmanathan cardiac electrophysiology

Bernabeu

Bordas

Cooper

Garny

Pitt-Francis

Whiteley

Gavaghan

58/10 Particle-scale structure in frozen colloidal suspensions from small angle X-ray scattering

Spannuth

Mochrie

Peppin

Wettlaufer

Copies of these, and any other OCCAM reports can be obtained from:

Oxford Centre for Collaborative Applied Mathematics Mathematical Institute 
24 - 29 St Giles'

Oxford

OX1 3LB

England

www.maths.ox.ac.uk/occam 